\title{
Adaptive Synchronization and Antisynchronization of a Hyperchaotic Complex Chen System with Unknown Parameters Based on Passive Control
}

\author{
Xiaobing Zhou, ${ }^{1}$ Lianglin Xiong, ${ }^{2}$ Weiwei Cai, ${ }^{1}$ and Xiaomei Cai ${ }^{3}$ \\ ${ }^{1}$ School of Information Science and Engineering, Yunnan University, Kunming 650091, China \\ ${ }^{2}$ School of Mathematics and Computer Science, Yunnan University of Nationalities, Kunming 650031, China \\ ${ }^{3}$ Bureau of Asset Management, Yunnan University, Kunming 650091, China
}

Correspondence should be addressed to Xiaobing Zhou; zhouxb.cn@gmail.com

Received 1 October 2012; Accepted 22 February 2013

Academic Editor: Erik Van Vleck

Copyright (c) 2013 Xiaobing Zhou et al. This is an open access article distributed under the Creative Commons Attribution License, which permits unrestricted use, distribution, and reproduction in any medium, provided the original work is properly cited.

This paper investigates the synchronization and antisynchronization problems of a hyperchaotic complex Chen system with unknown parameters based on the properties of a passive system. The essential conditions are derived under which the synchronization or antisynchronization error dynamical system could be equivalent to a passive system and be globally asymptotically stabilized at a zero equilibrium point via smooth state feedback. Corresponding parameter estimation update laws are obtained to estimate the unknown parameters as well. Numerical simulations verify the effectiveness of the theoretical analysis.

\section{Introduction}

Hyperchaos [1] is generally characterized as a chaotic attractor with more than one positive Lyapunov exponent and has richer dynamical behaviors than chaos. Over the past three decades, hyperchaotic systems with real variables have been investigated extensively [2-5]. Since Fowler et al. [6] introduced a complex Lorenz model to generalize the real Lorenz model in 1982, chaotic and hyperchaotic complex systems have attracted increasing attention to the systems with complex variables which can be used to describe the physics of a detuned laser, rotating fluids, disk dynamos, electronic circuits, and particle beam dynamics in high energy accelerators [7]. When applying the complex systems in communications, the complex variables will double the number of variables and can increase the content and security of the transmitted information.

In recent years, chaos synchronization has attracted increasing attention among scientists due to its potential applications in the fields of secure communications; optical, chemical, physical, and biological systems; neural networks; and so forth $[8,9]$. Several types of synchronization have been investigated on complex chaotic and hyperchaotic systems including complete synchronization [7], antisynchronization $[10,11]$, phase and antiphase synchronization [12], lag and antilag synchronization $[13,14]$, hybrid projective synchronization [15], and modified function projective synchronization [16]. Among the abovementioned synchronization phenomena, the most widely investigated one is complete synchronization (synchronization for short hereafter), which implies that the differences of state variables of synchronized systems starting from different initial values converge to zero eventually. On the other hand, antisynchronization is an another interesting phenomenon, which is characterized by the vanishing of the sum of the relevant state variables of synchronized systems. When applying antisynchronization to communication systems, the security and secrecy of communication can be strengthened while transmitting digital signals by the transform between synchronization and antisynchronization continuously.

Recently, many researchers have begun to give their attention to the concept of passivity of nonlinear systems. The passivity theory is considered to be an alternative tool for analyzing the stability of nonlinear systems. The main idea of passivity theory is that the passive properties of a system can keep the system internally stable. In order to make a system 
stable, one can design a controller which renders the closedloop system passive with the help of passivity theory. For the past decade, the passivity theory has played an important role in designing an asymptotically stabilizing controller for control and synchronization of chaotic and hyperchaotic systems with real variables [17-20]. For complex nonlinear systems, only Mahmoud et al. [21] applied the passive control to investigate the control of $n$-dimensional chaotic complex nonlinear systems. In this paper, we apply the passive control to investigate the synchronization and antisynchronization problems of the newly reported hyperchaotic complex Chen system [22].

This paper is organized as follows. Section 2 introduces the passive control theory and a new hyperchaotic complex Chen system. Sections 3 and 4 investigate the synchronization and antisynchronization problems of two identical hyperchaotic complex Chen systems with unknown parameters, respectively. In Section 5, two numerical examples are provided to illustrate the analytical results. Finally, conclusions are given in Section 6.

\section{The Passive Control Theory and a New Hyperchaotic Complex Chen System}

Consider the following nonlinear affine system:

$$
\begin{gathered}
\dot{x}=f(x)+g(x) u, \\
w=h(x),
\end{gathered}
$$

where $x \in R^{n}$ is the state variable and $u \in R^{m}$ and $w \in R^{m}$ are input and output values, respectively. $f(x)$ and $g(x)$ are smooth vector fields, $f(0)=0$, and $h(x)$ is a smooth mapping.

Definition 1 (see [23]). System (1) is a minimum phase system if $L_{g} h(0)$ is nonsingular and $x=0$ is one of the asymptotically stabilized equilibrium points of $f(x)$.

Definition 2 (see [24]). System (1) is passive if there exists a real constant $\beta$ such that for all $t \geq 0$, the following inequality holds:

$$
\int_{0}^{t} u^{T}(\tau) w(\tau) d \tau \geq \beta
$$

or there exists a $\rho \geq 0$ and a real constant $\beta$ such that

$$
\int_{0}^{t} u^{T}(\tau) w(\tau) d \tau+\beta \geq \int_{0}^{t} \rho w^{T}(\tau) w(\tau) d \tau .
$$

If system (1) has relative degree $[1, \ldots, 1]$ at $x=0$ (i.e., $L_{g} h(0)$ is nonsingular) and the distribution spanned by the vector field $g_{1}(x), \ldots, g_{m}(x)$ is innovative, then it can be represented as the following normal form:

$$
\begin{gathered}
\dot{z}=f_{0}(z)+p(z, w) w, \\
\dot{w}=b(z, w)+a(z, w) u,
\end{gathered}
$$

where $a(z, w)$ is nonsingular for any $(z, w)$.
Theorem 3 (see [24]). Suppose the system (4) is passive with a storage function $V$, which is positive-definite, and the system (4) is locally zero-state detectable. Let $\phi$ be a smooth function such that $\phi(0)=0$ and $w^{T} \phi(w)>0$ for each nonzero $w$. Then the control law $u=-\phi(w)$ asymptotically stabilizes the equilibrium of system (4).

Recently, the authors [22] introduced six different versions of the hyperchaotic complex Chen system by adding a state feedback controller to the chaotic complex Chen system [25], and the dynamics of the six hyperchaotic complex Chen systems were studied in detail in [22]. In this paper, we apply the passive control to investigate the synchronization and antisynchronization problems of the following form of hyperchaotic complex Chen system:

$$
\begin{gathered}
\dot{x}=\alpha(y-x)+w, \\
\dot{y}=(\gamma-\alpha) x-x z+\gamma y, \\
\dot{z}=\frac{1}{2}(\bar{x} y+x \bar{y})-\beta z+w, \\
\dot{w}=\frac{1}{2}(\bar{x} y+x \bar{y})-d w,
\end{gathered}
$$

where $\alpha, \beta, \gamma$, and $d$ are positive real parameters, $x=u_{1}+i u_{2}$ and $y=u_{3}+i u_{4}$ are complex functions, $i=\sqrt{-1}, z=u_{5}$, and $w=u_{6} \cdot u_{j}(j=1,2,3,4,5,6)$ are real functions. The overbar represents complex conjugate function.

\section{Synchronization of the Hyperchaotic Complex Chen System with Unknown Parameters}

In this section, we study the synchronization problem of the hyperchaotic complex Chen system (5) with unknown parameters using the technique of passive control. We consider system (5) as the drive system, and the response system is described by

$$
\begin{gathered}
\dot{x}_{1}=\alpha\left(y_{1}-x_{1}\right)+w_{1}, \\
\dot{y}_{1}=(\gamma-\alpha) x_{1}-x_{1} z_{1}+\gamma y_{1}+\mu_{1}+i \mu_{2}, \\
\dot{z}_{1}=\frac{1}{2}\left(\bar{x}_{1} y_{1}+x_{1} \bar{y}_{1}\right)-\beta z_{1}+w_{1}+\mu_{3}, \\
\dot{w}_{1}=\frac{1}{2}\left(\bar{x}_{1} y_{1}+x_{1} \bar{y}_{1}\right)-d w_{1}+\mu_{4},
\end{gathered}
$$

where $x_{1}=v_{1}+i v_{2}, y_{1}=v_{3}+i v_{4}$, and $z_{1}=v_{5}, w_{1}=v_{6}$. $v_{j}(j=1,2,3,4,5,6)$ are real functions, and $\mu_{1}, \mu_{2}, \mu_{3}$, and $\mu_{4}$ are real control functions to be determined to achieve synchronization. 
By subtracting the drive system (5) from the response system (6), we obtain the following error dynamical system

$$
\begin{aligned}
& \dot{e}_{1}+i \dot{e}_{2}=\alpha\left(y_{1}-x_{1}\right)+w_{1}-(\alpha(y-x)+w), \\
& \dot{e}_{3}+i \dot{e}_{4}=(\gamma-\alpha) x_{1}-x_{1} z_{1}+\gamma y_{1} \\
&-((\gamma-\alpha) x-x z+\gamma y)+\mu_{1}+i \mu_{2}, \\
& \dot{e}_{5}=\frac{1}{2}\left(\bar{x}_{1} y_{1}+x_{1} \bar{y}_{1}\right)-\beta z_{1}+w_{1} \\
& \quad\left(\frac{1}{2}(\bar{x} y+x \bar{y})-\beta z+w\right)+\mu_{3}, \\
& \dot{e}_{6}=\frac{1}{2}\left(\bar{x}_{1} y_{1}+x_{1} \bar{y}_{1}\right)-d w_{1}-\left(\frac{1}{2}(\bar{x} y+x \bar{y})-d w\right)+\mu_{4},
\end{aligned}
$$

where $e_{j}=v_{j}-u_{j}(j=1,2,3,4,5,6)$ are error states and $\alpha$, $\beta, \gamma$, and $d$ are unknown parameters.

Separating the real and imaginary parts of error dynamical system (7), we obtain the following real system:

$$
\begin{aligned}
& \dot{e}_{1}=\alpha\left(e_{3}-e_{1}\right)+e_{6}, \\
& \dot{e}_{2}=\alpha\left(e_{4}-e_{2}\right), \\
& \dot{e}_{3}=(\gamma-\alpha) e_{1}+\gamma e_{3}-e_{1} e_{5}-e_{1} u_{5}-u_{1} e_{5}+\mu_{1}, \\
& \dot{e}_{4}=(\gamma-\alpha) e_{2}+\gamma e_{4}-e_{2} e_{5}-e_{2} u_{5}-u_{2} e_{5}+\mu_{2}, \\
& \dot{e}_{5}=e_{1} e_{3}+e_{1} u_{3}+u_{1} e_{3}+e_{2} e_{4}+e_{2} u_{4}+u_{2} e_{4}-\beta e_{5}+e_{6}+\mu_{3}, \\
& \dot{e}_{6}=e_{1} e_{3}+e_{1} u_{3}+u_{1} e_{3}+e_{2} e_{4}+e_{2} u_{4}+u_{2} e_{4}-d e_{6}+\mu_{4} .
\end{aligned}
$$

Let $z_{1}=e_{1}, z_{2}=e_{2}, y_{1}=e_{3}, y_{2}=e_{4}, y_{3}=e_{5}$, and $y_{4}=e_{6}$; then the error dynamical system (8) can be rewritten as

$$
\begin{aligned}
& \dot{z}_{1}=\alpha\left(y_{1}-z_{1}\right)+y_{4}, \\
& \dot{z}_{2}=\alpha\left(y_{2}-z_{2}\right), \\
& \dot{y}_{1}=(\gamma-\alpha) z_{1}+\gamma y_{1}-z_{1} y_{3}-z_{1} u_{5}-u_{1} y_{3}+\mu_{1}, \\
& \dot{y}_{2}=(\gamma-\alpha) z_{2}+\gamma y_{2}-z_{2} y_{3}-z_{2} u_{5}-u_{2} y_{3}+\mu_{2}, \\
& \dot{y}_{3}=z_{1} y_{1}+z_{1} u_{3}+u_{1} y_{1}+z_{2} y_{2}+z_{2} u_{4}+u_{2} y_{2}-\beta y_{3} \\
& \quad \quad+y_{4}+\mu_{3}, \\
& \dot{y}_{4}=z_{1} y_{1}+z_{1} u_{3}+u_{1} y_{1}+z_{2} y_{2}+z_{2} u_{4}+u_{2} y_{2}-d y_{4}+\mu_{4},
\end{aligned}
$$

which is the following normal form:

$$
\begin{aligned}
& \dot{z}=f_{0}(z)+p(z, y) y, \\
& \dot{y}=b(z, y)+a(z, y) \mu,
\end{aligned}
$$

where $z=\left[z_{1}, z_{2}\right]^{T}, y=\left[y_{1}, y_{2}, y_{3}, y_{4}\right]^{T}, \mu=\left[\mu_{1}, \mu_{2}, \mu_{3}, \mu_{4}\right]^{T}$ and

$$
\begin{aligned}
& f_{0}(z)=\left[-\alpha z_{1},-\alpha z_{2}\right]^{T}, \quad p(z, y)=\left[\begin{array}{llll}
\alpha & 0 & 0 & 1 \\
0 & \alpha & 0 & 0
\end{array}\right], \\
& a(z, y)=\left[\begin{array}{llll}
1 & 0 & 0 & 0 \\
0 & 1 & 0 & 0 \\
0 & 0 & 1 & 0 \\
0 & 0 & 0 & 1
\end{array}\right], \\
& b(z, y)= \\
& {\left[\begin{array}{c}
(\gamma-\alpha) z_{1}+\gamma y_{1}-z_{1} y_{3}-z_{1} u_{5}-u_{1} y_{3} \\
(\gamma-\alpha) z_{2}+\gamma y_{2}-z_{2} y_{3}-z_{2} u_{5}-u_{2} y_{3} \\
z_{1} y_{1}+z_{1} u_{3}+u_{1} y_{1}+z_{2} y_{2}+z_{2} u_{4}+u_{2} y_{2}-\beta y_{3}+y_{4} \\
z_{1} y_{1}+z_{1} u_{3}+u_{1} y_{1}+z_{2} y_{2}+z_{2} u_{4}+u_{2} y_{2}-d y_{4}
\end{array}\right] .}
\end{aligned}
$$

Then we can arrive at the following result.

Theorem 4. If the passive controllers are designed as

$$
\begin{aligned}
& \mu_{1}=-\tilde{\gamma} z_{1}-\tilde{\gamma} y_{1}+z_{1} y_{3}+z_{1} u_{5}+u_{1} y_{3}-k_{1} y_{1}+v_{1}, \\
& \mu_{2}=-\tilde{\gamma} z_{2}-\tilde{\gamma} y_{2}+z_{2} y_{3}+z_{2} u_{5}+u_{2} y_{3}-k_{2} y_{2}+v_{2} \text {, } \\
& \mu_{3}=-z_{1} y_{1}-z_{1} u_{3}-u_{1} y_{1}-z_{2} y_{2}-z_{2} u_{4}-u_{2} y_{2}+\tilde{\beta} y_{3} \\
& -y_{4}-k_{3} y_{3}+v_{3} \\
& \mu_{4}=-z_{1} y_{1}-z_{1} u_{3}-u_{1} y_{1}-z_{2} y_{2}-z_{2} u_{4}-u_{2} y_{2}+\tilde{d} y_{4}-z_{1} \\
& -k_{4} y_{4}+v_{4}
\end{aligned}
$$

and the parameter estimation update laws as

$$
\begin{gathered}
\tilde{\beta}=-y_{3}^{2}, \\
\tilde{\gamma}=z_{1} y_{1}+y_{1}^{2}+z_{2} y_{2}+y_{2}^{2}, \\
\tilde{d}=-y_{4}^{2},
\end{gathered}
$$

where $k=\left[k_{1}, k_{2}, k_{3}, k_{4}\right]^{T}$ is a positive constant vector, $v=$ $\left[v_{1}, v_{2}, v_{3}, v_{4}\right]^{T}$ is an external signal vector which is connected with the reference input, and $\tilde{\beta}, \tilde{\gamma}$, and $\tilde{d}$ are estimated values of the unknown parameters $\alpha, \beta, \gamma$, and $d$, respectively, then the error dynamical system (8) will be rendered passive and will be asymptotically stable at its equilibrium $(0,0,0,0)$ and the two systems (5) and (6) starting from different initial values will be synchronized.

Proof. Construct the following storage function:

$$
\begin{aligned}
V(z, y)= & W(z)+\frac{1}{2} y^{T} y+\frac{1}{2}(\widetilde{\alpha}-\alpha)^{2}+\frac{1}{2}(\widetilde{\beta}-\beta)^{2} \\
& +\frac{1}{2}(\widetilde{\gamma}-\gamma)^{2}+\frac{1}{2}(\widetilde{d}-d)^{2},
\end{aligned}
$$

where $W(z)=(1 / 2)\left(z_{1}^{2}+z_{2}^{2}\right)$ is a Lyapunov function of $f_{0}(z)$. 


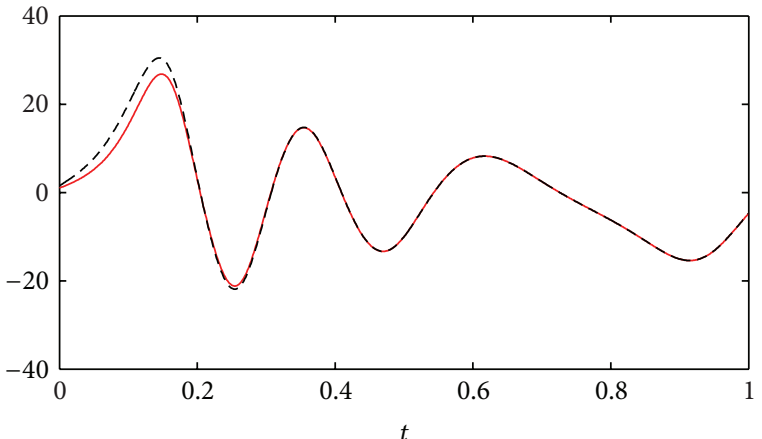

$-u_{1}$

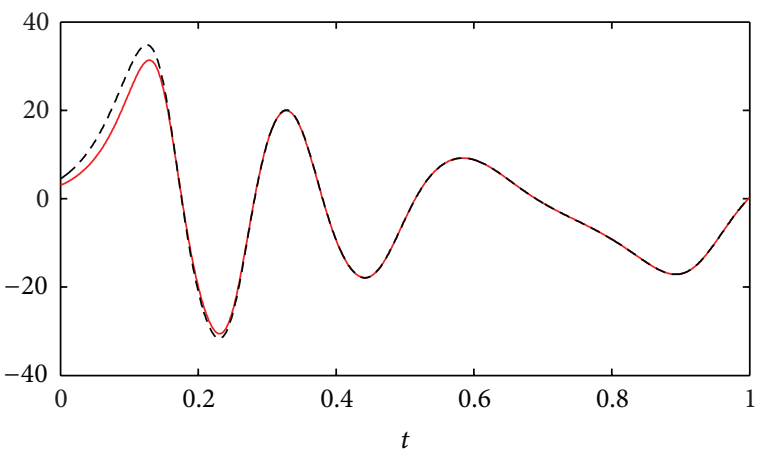

$-u_{3}$

$---v_{3}$

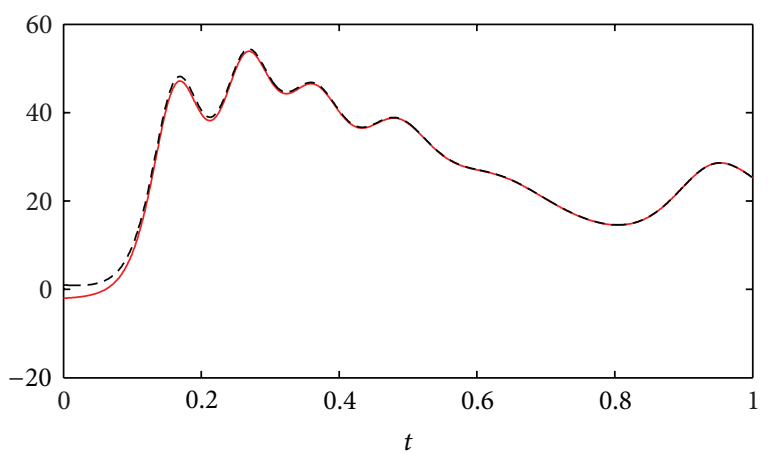

$-u_{5}$
$---v_{5}$

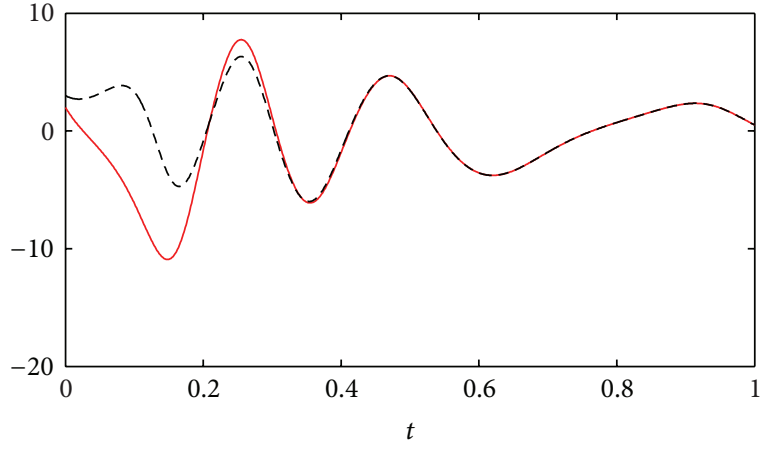

$-u_{2}$

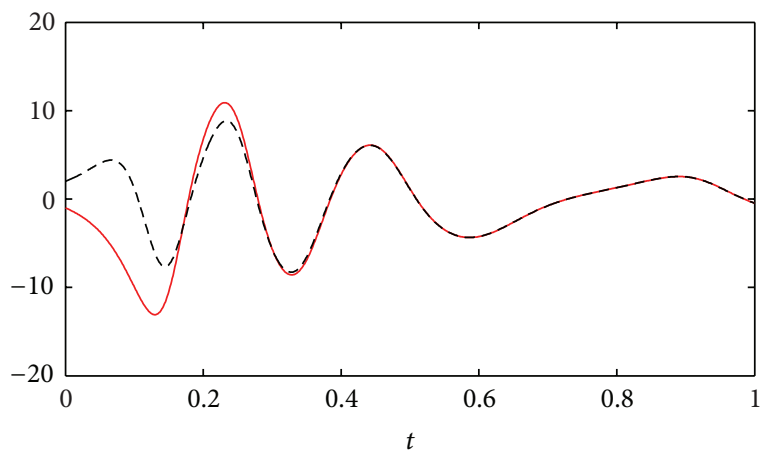

$-u_{4}$

$---v_{4}$

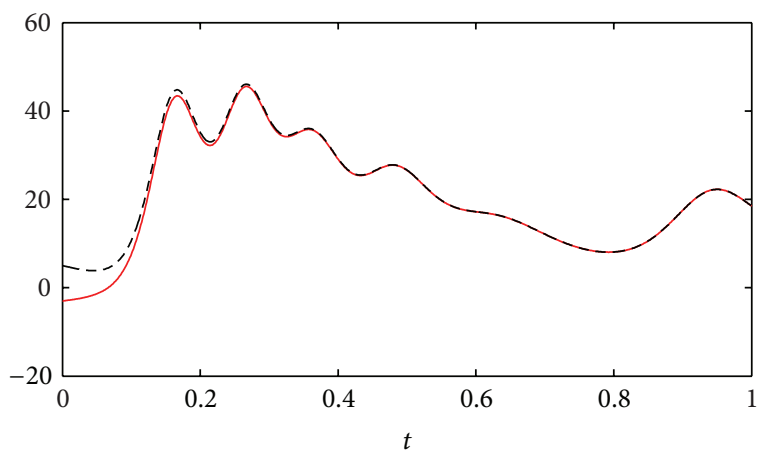

$-u_{6}$

FIGURE 1: The time response of states for the drive system (5) and the response system (6) with controllers (12) and parameter estimation update laws (13).

The zero dynamics of system (10) describe the internal dynamics, which are consistent with the external constraint $y=0$; that is, $\dot{z}=f_{0}(z)$.

Differentiating $W(z)$ with respect to $t$, we have

$$
\frac{d}{d t} W(z)=\frac{\partial W(z)}{\partial z} f_{0}(z)=-\alpha z_{1}^{2}-\alpha z_{2}^{2} \leq 0
$$

Then $f_{0}(z)$ is globally asymptotically stable; that is, the zero dynamics of system (8) is Lyapunov stable. In the light of Definition 1, system (8) is a minimum phase system.
Furthermore, taking the time derivative of $V(z, y)$ along the trajectory of the error dynamical system (8) yields

$$
\begin{aligned}
\frac{d}{d t} V(z, y)= & \frac{\partial W(z)}{\partial z} \dot{z}+y^{T} \dot{y}+(\widetilde{\alpha}-\alpha) \dot{\tilde{\alpha}}+(\widetilde{\beta}-\beta) \dot{\tilde{\beta}} \\
& +(\widetilde{\gamma}-\gamma) \dot{\tilde{\gamma}}+(\widetilde{d}-d) \dot{\tilde{d}} \\
= & \frac{\partial W(z)}{\partial z} f_{0}(z)+\frac{\partial W(z)}{\partial z} p(z, y) y \\
& +y^{T} b(z, y)+y^{T} a(z, y) \mu+(\widetilde{\alpha}-\alpha) \dot{\tilde{\alpha}} \\
& +(\widetilde{\beta}-\beta) \dot{\tilde{\beta}}+(\widetilde{\gamma}-\gamma) \dot{\tilde{\gamma}}+(\widetilde{d}-d) \dot{\tilde{d}} .
\end{aligned}
$$


Since the error dynamical system (8) is a minimum phase system; that is, $((\partial W(z)) / \partial z) f_{0}(z) \leq 0$, then (15) becomes

$$
\begin{aligned}
\frac{d}{d t} V(z, y) \leq & \frac{\partial W(z)}{\partial z} p(z, y) y+y^{T} b(z, y)+y^{T} a(z, y) \mu \\
& +(\widetilde{\alpha}-\alpha) \dot{\tilde{\alpha}}+(\widetilde{\beta}-\beta) \dot{\tilde{\beta}}+(\widetilde{\gamma}-\gamma) \dot{\tilde{\gamma}} \\
& +(\widetilde{d}-d) \dot{\tilde{d}} .
\end{aligned}
$$

Substituting (12) and (13) into (17) yields

$$
\frac{d}{d t} V(z, y) \leq-k y^{T} y+v^{T} y
$$

Then, taking integration on both sides of (18), we get

$$
\begin{aligned}
V(z, y)-V\left(z_{0}, y_{0}\right) \leq & -\int_{0}^{t} k y^{T}(\tau) y(\tau) d \tau \\
& +\int_{0}^{t} \nu^{T}(\tau) y(\tau) d \tau .
\end{aligned}
$$

For $V(z, y) \geq 0$, let $V\left(z_{0}, y_{0}\right)=u$; then the above inequality can be rewritten as

$$
\begin{aligned}
\int_{0}^{t} v^{T}(\tau) y(\tau) d \tau+u & \geq \int_{0}^{t} k y^{T}(\tau) y(\tau) d \tau+V(z, y) \\
& \geq \int_{0}^{t} k y^{T}(\tau) y(\tau) d \tau .
\end{aligned}
$$

Letting $v=\left[v_{1}, v_{2}, v_{3}, v_{4}\right]^{T}=[0,0,0,0]^{T}$, in light of Definition 2 and Theorem 3 , system (8) will be stabilized at its equilibrium $(0,0,0,0)$ with the controllers (12) and the parameter estimation update laws (13); that is, the drive system (5) and response system (6) with different initial conditions will be synchronized with each other asymptotically.

This completes the proof.

Remark 5. The controllers (12) are only related to the parameters $\beta, \gamma$, and $d$, and so we do not need to estimate the parameter $\alpha$.

\section{Antisynchronization of the Hyperchaotic Complex Chen System with Unknown Parameters}

To investigate the antisynchronization of the hyperchaotic complex Chen system, we need to add system (6) to system (5) and obtain the following antisynchronization error dynamical system:

$$
\dot{e}_{1}+i \dot{e}_{2}=\alpha\left(y_{1}-x_{1}\right)+w_{1}+(\alpha(y-x)+w),
$$

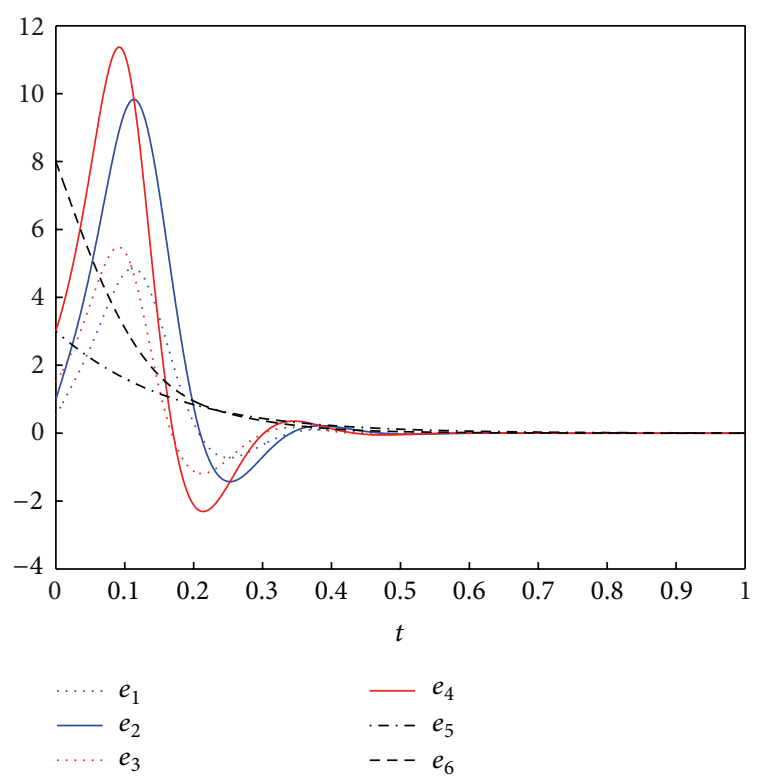

FIGURE 2: The time response of synchronization error states $e_{j}(j=$ $1,2,3,4,5,6)$ for the error dynamical system (8).

$$
\begin{aligned}
\dot{e}_{3}+i \dot{e}_{4}=(\gamma-\alpha) x_{1}-x_{1} z_{1}+\gamma y_{1}+((\gamma-\alpha) x-x z+\gamma y) & \\
& +\mu_{1}+i \mu_{2}, \\
\dot{e}_{5}= & \frac{1}{2}\left(\bar{x}_{1} y_{1}+x_{1} \bar{y}_{1}\right)-\beta z_{1}+w_{1} \\
& +\left(\frac{1}{2}(\bar{x} y+x \bar{y})-\beta z+w\right)+\mu_{3}, \\
\dot{e}_{6}= & \frac{1}{2}\left(\bar{x}_{1} y_{1}+x_{1} \bar{y}_{1}\right)-d w_{1}+\left(\frac{1}{2}(\bar{x} y+x \bar{y})-d w\right)+\mu_{4},
\end{aligned}
$$

where $e_{j}=v_{j}+u_{j}(j=1,2,3,4,5,6)$ are antisynchronization error states and $\alpha, \beta, \gamma$, and $d$ are unknown parameters. By separating the real and imaginary parts of the antisynchronization error dynamical system (21), we get the following real system:

$$
\begin{aligned}
& \dot{e}_{1}=\alpha\left(e_{3}-e_{1}\right)+e_{6}, \\
& \dot{e}_{2}=\alpha\left(e_{4}-e_{2}\right), \\
& \dot{e}_{3}=(\gamma-\alpha) e_{1}+\gamma e_{3}-e_{1} e_{5}+e_{1} u_{5}+u_{1} e_{5}-2 u_{1} u_{5}+\mu_{1}, \\
& \dot{e}_{4}=(\gamma-\alpha) e_{2}+\gamma e_{4}-e_{2} e_{5}+e_{2} u_{5}+u_{2} e_{5}-2 u_{2} u_{5}+\mu_{2}, \\
& \dot{e}_{5}=e_{1} e_{3}-e_{1} u_{3}-u_{1} e_{3}+2 u_{1} u_{3}+e_{2} e_{4}-e_{2} u_{4}-u_{2} e_{4} \\
&+2 u_{2} u_{4}-\beta e_{5}+e_{6}+\mu_{3}, \\
& \dot{e}_{6}= e_{1} e_{3}-e_{1} u_{3}-u_{1} e_{3}+2 u_{1} u_{3}+e_{2} e_{4}-e_{2} u_{4}-u_{2} e_{4} \\
&+2 u_{2} u_{4}-d e_{6}+\mu_{4} .
\end{aligned}
$$




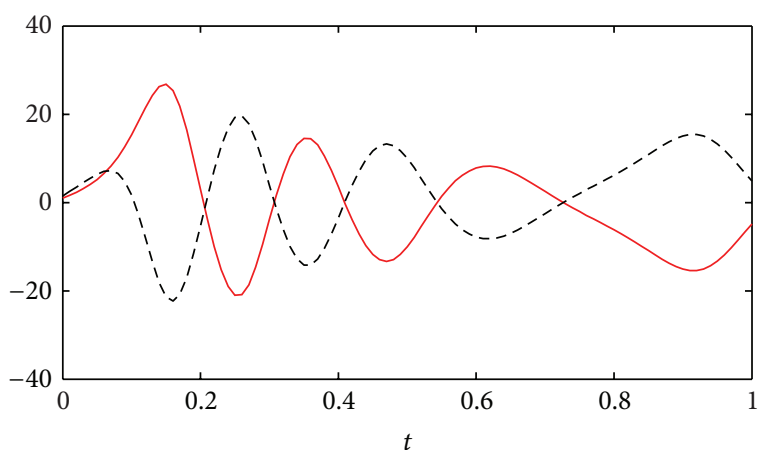

$-u_{1}$

$---v_{1}$

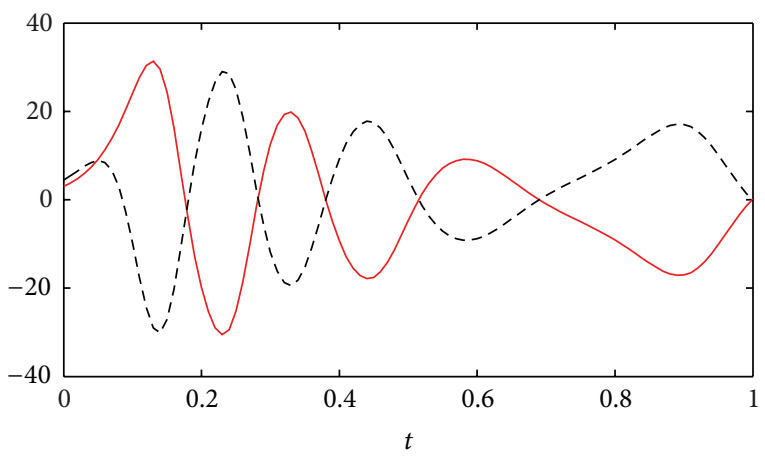

$-u_{3}$

-- $v_{3}$

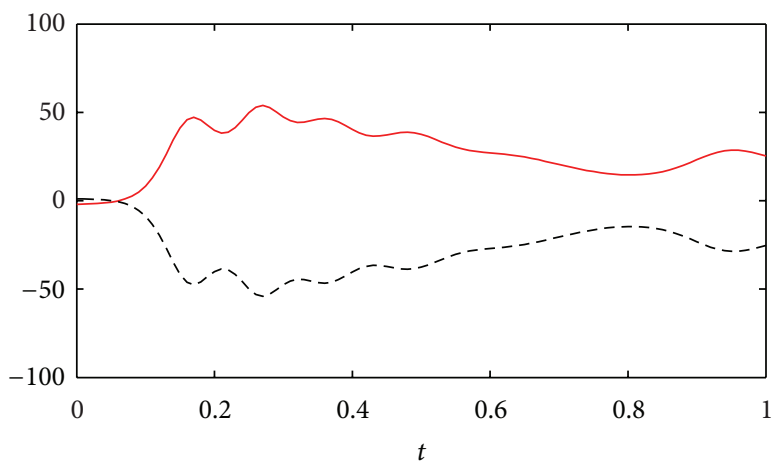

$\begin{array}{ll}- & u_{5} \\ --- & v_{5}\end{array}$

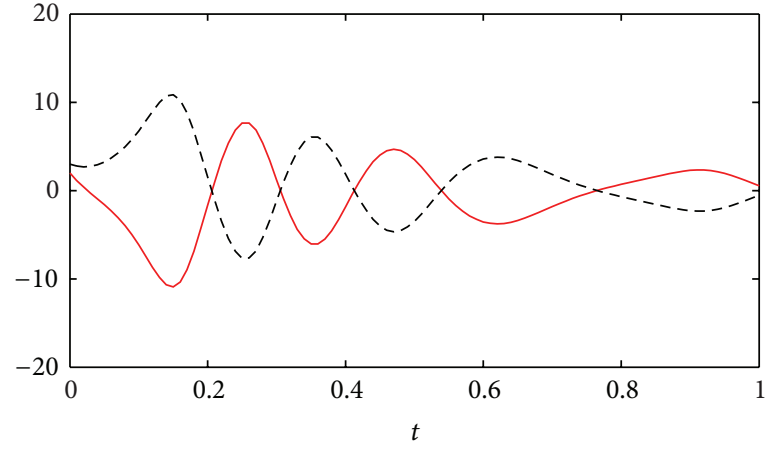

$-u_{2}$

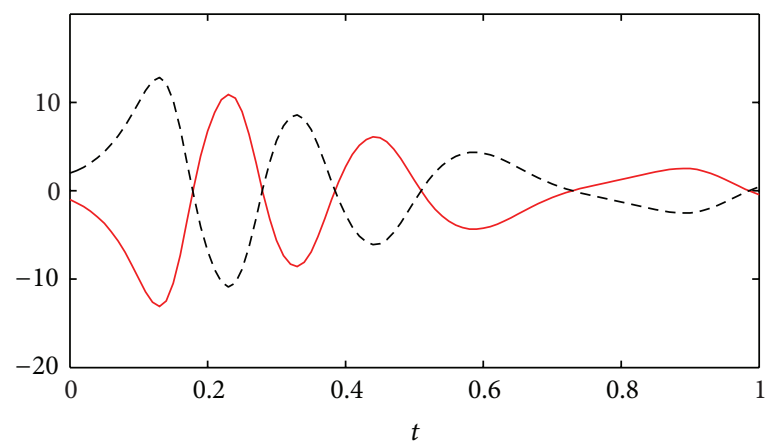

$-u_{4}$

-- $v_{4}$

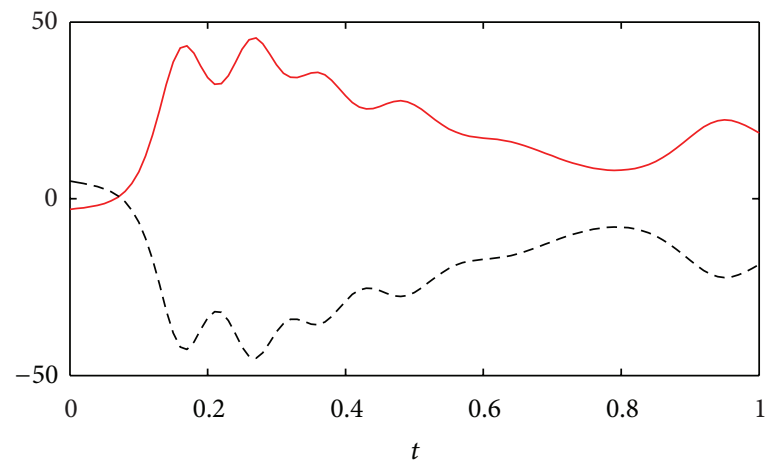

$-u_{6}$

FIGURE 3: The time response of states for the drive system (5) and the response system (6) with controllers (24) and parameter estimation update laws (25).

Let $z_{1}=e_{1}, z_{2}=e_{2}, y_{1}=e_{3}, y_{2}=e_{4}, y_{3}=e_{5}$, and $y_{4}=e_{6}$; then the error dynamical system (22) can be rewritten as

$$
\begin{aligned}
\dot{z}_{1}= & \alpha\left(y_{1}-z_{1}\right)+y_{4}, \\
\dot{z}_{2}= & \alpha\left(y_{2}-z_{2}\right), \\
\dot{y}_{1}= & (\gamma-\alpha) z_{1}+\gamma y_{1}-z_{1} y_{3}+z_{1} u_{5}+u_{1} y_{3}-2 u_{1} u_{5}+\mu_{1}, \\
\dot{y}_{2}= & (\gamma-\alpha) z_{2}+\gamma y_{2}-z_{2} y_{3}+z_{2} u_{5}+u_{2} y_{3}-2 u_{2} u_{5}+\mu_{2}, \\
\dot{y}_{3}= & z_{1} y_{1}-z_{1} u_{3}-u_{1} y_{1}+2 u_{1} u_{3}+z_{2} y_{2}-z_{2} u_{4}-u_{2} y_{2} \\
& +2 u_{2} u_{4}-\beta y_{3}+y_{4}+\mu_{3},
\end{aligned}
$$

$$
\begin{aligned}
\dot{y}_{4}= & z_{1} y_{1}-z_{1} u_{3}-u_{1} y_{1}+2 u_{1} u_{3}+z_{2} y_{2}-z_{2} u_{4}-u_{2} y_{2} \\
& +2 u_{2} u_{4}-d y_{4}+\mu_{4} .
\end{aligned}
$$

Thus, we can establish the following theorem.

Theorem 6. If the passive controllers are designed as

$$
\begin{aligned}
\mu_{1}= & -\tilde{\gamma} z_{1}-\tilde{\gamma} y_{1}+z_{1} y_{3}-z_{1} u_{5}-u_{1} y_{3}+2 u_{1} u_{5}-k_{1} y_{1}+v_{1}, \\
\mu_{2}= & -\tilde{\gamma} z_{2}-\tilde{\gamma} y_{2}+z_{2} y_{3}-z_{2} u_{5}-u_{2} y_{3}+2 u_{2} u_{5}-k_{2} y_{2}+v_{2}, \\
\mu_{3}= & -z_{1} y_{1}+z_{1} u_{3}+u_{1} y_{1}-2 u_{1} u_{3}-z_{2} y_{2}+z_{2} u_{4}+u_{2} y_{2} \\
& -2 u_{2} u_{4}+\tilde{\beta} y_{3}-y_{4}-k_{3} y_{3}+v_{3},
\end{aligned}
$$




$$
\begin{aligned}
\mu_{4}= & -z_{1} y_{1}+z_{1} u_{3}+u_{1} y_{1}-2 u_{1} u_{3}-z_{2} y_{2}+z_{2} u_{4}+u_{2} y_{2} \\
& -2 u_{2} u_{4}+\tilde{d} y_{4}-z_{1}-k_{4} y_{4}+v_{4}
\end{aligned}
$$

and the parameter estimation update laws as

$$
\begin{gathered}
\widetilde{\beta}=-y_{3}^{2}, \\
\widetilde{\gamma}=z_{1} y_{1}+y_{1}^{2}+z_{2} y_{2}+y_{2}^{2}, \\
\widetilde{d}=-y_{4}^{2},
\end{gathered}
$$

where $k=\left[k_{1}, k_{2}, k_{3}, k_{4}\right]^{T}$ is a positive constant vector, $v=$ $\left[v_{1}, v_{2}, v_{3}, v_{4}\right]^{T}$ is an external signal vector which is connected with the reference input, and $\tilde{\beta}, \tilde{\gamma}$, and $\widetilde{d}$ are estimated values of the unknown parameters $\beta, \gamma$, and $d$, respectively, then the antisynchronization error dynamical system (22) will be rendered passive and will be asymptotically stable at its equilibrium $(0,0,0,0)$ and the two systems (5) and (6) starting from different initial values will be antisynchronized.

Proof. The proof of Theorem 6 is similar to that of Theorem 4, so it is omitted here.

\section{Numerical Simulations}

In this section, we perform two numerical simulations to demonstrate the effectiveness of the above synchronization and antisynchronization schemes. In the following numerical simulations, the fourth-order Runge-Kutta method is used to solve the systems with time step size 0.001 . The system parameters are selected as $\alpha=32, \beta=5, \gamma=25$, and $d=6$ so that the hyperchaotic complex Chen system (5) exhibits hyperchaos.

Example 7. For the synchronization of the hyperchaotic complex Chen system, we consider the drive system (5) and the response system (6) with the controllers (12) and update laws (13). The initial values for the drive system (5) and response system (6) are given as $(x(0), y(0), z(0), w(0))=(1+2 i, 3-$ $i,-2,-3)$ and $\left(x_{1}(0), y_{1}(0), z_{1}(0), w_{1}(0)\right)=(1.5+3 i, 4.5+$ $2 i, 1,5)$; thus $\left(u_{1}, u_{2}, u_{3}, u_{4}, u_{5}, u_{6}\right)=(1,2,3,-1,-2,-3)$ and $\left(v_{1}, v_{2}, v_{3}, v_{4}, v_{5}, v_{6}\right)=(1.5,3,4.5,2,1,5)$, respectively. The initial errors are $\left(e_{1}, e_{2}, e_{3}, e_{4}, e_{5}, e_{6}\right)=(0.5,1,1.5,3,3,8)$. And the initial values of the parameter estimation update laws are $\widetilde{\beta}(0)=\widetilde{\gamma}(0)=\widetilde{d}(0)=0.1$. We choose $k_{1}=k_{2}=$ $k_{3}=k_{4}=1$ and $v_{1}=v_{2}=v_{3}=v_{4}=0$. Figure 1 shows the time response of states determined by the drive system (5) and the response system (6) with the controllers (12) and the parameter estimation update laws (13). Figure 2 shows the time response of error states for the error dynamical system (8). From Figures 1 and 2, we can see that the two systems (5) and (6) starting from different initial conditions synchronize with each other immediately and the trajectories of the error dynamical system (8) are asymptotically stabilized at the equilibrium point $O(0,0,0,0)$.

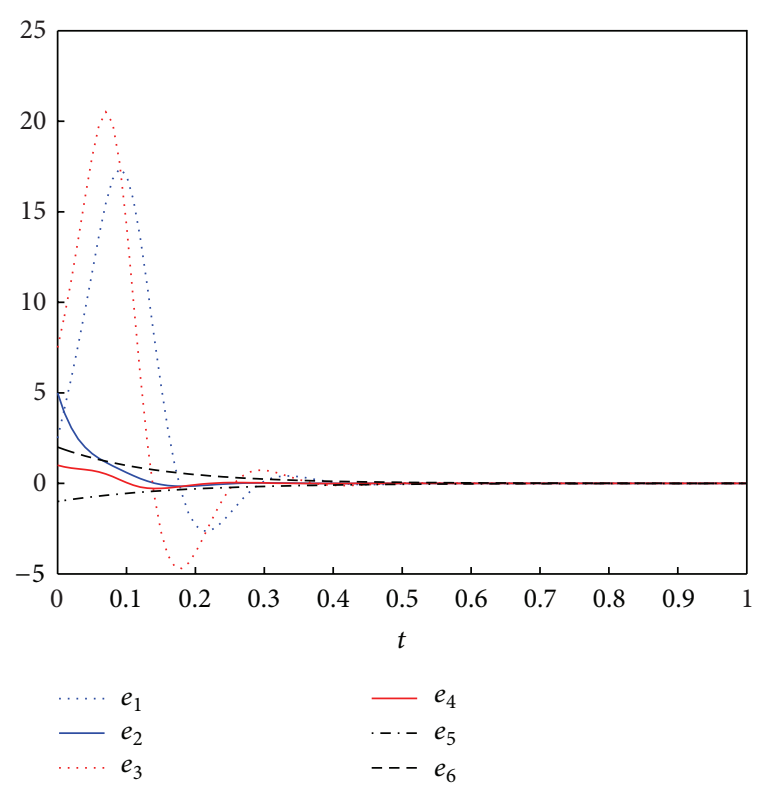

FIGURE 4: The time response of antisynchronization error states $e_{j}(j=1,2,3,4,5,6)$ for the error dynamical system (22).

Example 8. For the antisynchronization of the hyperchaotic complex Chen system, we also consider the drive system (5) and the response system (6) but with the controllers (24) and update laws (25). The initial values for the drive system (5) and response system (6) are also given as $(x(0), y(0), z(0), w(0))=(1+2 i, 3-i$, $-2,-3)$ and $\left(x_{1}(0), y_{1}(0), z_{1}(0), w_{1}(0)\right)=(1.5+3 i, 4.5+$ $2 i, 1,5)$; thus $\left(u_{1}, u_{2}, u_{3}, u_{4}, u_{5}, u_{6}\right)=(1,2,3,-1,-2,-3)$ and $\left(v_{1}, v_{2}, v_{3}, v_{4}, v_{5}, v_{6}\right)=(1.5,3,4.5,2,1,5)$, respectively. But the initial errors are $\left(e_{1}, e_{2}, e_{3}, e_{4}, e_{5}, e_{6}\right)=(2.5,5,7.5,1,-1,2)$. And the initial values of the parameter estimation update laws are $\widetilde{\beta}(0)=\tilde{\gamma}(0)=\tilde{d}(0)=0.1$. We choose $k_{1}=k_{2}=$ $k_{3}=k_{4}=1$ and $v_{1}=v_{2}=v_{3}=v_{4}=0$. Figure 3 shows the time response of states determined by the drive system (5) and the response system (6) with the controllers (24) and the parameter estimation update laws (25). The time response of error states for the antisynchronization error dynamical system (22) is shown in Figure 4. From Figures 3 and 4 , we can see that the two systems starting from different initial conditions antisynchronize with each other immediately and the trajectories of the antisynchronization error dynamical system (22) are asymptotically stabilized at the zero equilibrium.

\section{Conclusions}

Hyperchaotic systems with real variables have been investigated extensively over the past three decades. But hyperchaotic complex systems have attracted increasing attention due to the fact that they have much wider applications. So we investigate the synchronization and antisynchronization problems of a hyperchaotic complex Chen system by applying the passive control technique. Based on the fact that once a 
system is passive, there exists a control law that makes the passive system stable, then the passivity-based controller can be proposed to asymptotically stabilize the error dynamical system. Then corresponding passive controllers and update laws of the parameters are proposed to achieve synchronization and antisynchronization between two hyperchaotic complex Chen systems with different initial conditions, respectively. Furthermore, this work can be extended to achieve synchronization and antisynchronization of other versions of the hyperchaotic complex Chen system, even other types of hyperchaotic complex systems, such as Lorenz system [26] and Lü system [27].

\section{Acknowledgments}

This work was supported by the Youth Foundation of Yunnan University of Nationalities under Grant no. 11QN07, the Natural Science Foundation of Yunnan Province under Grants no. 2009CD019 and no. 2011FZ172, and the Natural Science Foundation of China under Grants no. 61065008, no. 61005087, and no. 61263042 .

\section{References}

[1] O. E. Rössler, "An equation for hyperchaos," Physics Letters A, vol. 71, no. 2-3, pp. 155-157, 1979.

[2] T. Matsumoto, L. O. Chua, and K. Kobayashi, "Hyperchaos: laboratory experiment and numerical confirmation," IEEETransactions on Circuits and Systems, vol. 33, no. 11, pp. 1143$1149,1986$.

[3] G. Grassi and S. Mascolo, "A system theory approach for designing cryptosystems based on hyperchaos," IEEE Transactions on Circuits and Systems I-Fundamental Theory and Applications, vol. 46, no. 9, pp. 1135-1138, 1999.

[4] H. Yin, Z. Chen, and Z. Yuan, "A blind watermarking algorithm based on hyperchaos and coset by quantizing wavelet transform coefficients," International Journal of Innovative Computing, Information and Control, vol. 3, no. 6 B, pp. 1635-1643, 2007.

[5] C. X. Zhu, "A novel image encryption scheme based on improved hyperchaotic sequences," Optics Communications, vol. 285, no. 1, pp. 29-37, 2012.

[6] A. C. Fowler, M. J. McGuinness, and J. D. Gibbon, "The complex Lorenz equations,” Physica D, vol. 4, no. 2, pp. 139-163, 1982.

[7] G. M. Mahmoud and E. E. Mahmoud, "Complete synchronization of chaotic complex nonlinear systems with uncertain parameters," Nonlinear Dynamics, vol. 62, no. 4, pp. 875-882, 2010.

[8] L. M. Pecora and T. L. Carroll, "Synchronization in chaotic systems," Physical Review Letters, vol. 64, no. 8, pp. 821-824, 1990.

[9] S. Boccaletti, J. Kurths, G. Osipov, D. L. Valladares, and C. S. Zhou, "The synchronization of chaotic systems," Physics Reports A, vol. 366, no. 1-2, pp. 1-101, 2002.

[10] S. Liu and P. Liu, "Adaptive anti-synchronization of chaotic complex nonlinear systems with unknown parameters," Nonlinear Analysis, vol. 12, no. 6, pp. 3046-3055, 2011.

[11] P. Liu and S. T. Liu, "Anti-synchronization between different chaotic complex systems," Physica Scripta, vol. 83, no. 6, Article ID 065006, 2011.
[12] G. M. Mahmoud and E. E. Mahmoud, "Phase and antiphase synchronization of two identical hyperchaotic complex nonlinear systems," Nonlinear Dynamics, vol. 61, no. 1-2, pp. 141-152, 2010.

[13] G. M. Mahmoud and E. E. Mahmoud, "Lag synchronization of hyperchaotic complex nonlinear systems," Nonlinear Dynamics, vol. 67, no. 2, pp. 1613-1622, 2012.

[14] E. E. Mahmoud, "Adaptive anti-lag synchronization of two identical or non-identical hyperchaotic complex nonlinear systems with uncertain parameters," Journal of the Franklin Institute-Engineering and Applied Mathematics, vol. 349, no. 3, pp. 1247-1266, 2012.

[15] M. Hu, Y. Yang, Z. Xu, and L. Guo, "Hybrid projective synchronization in a chaotic complex nonlinear system," Mathematics and Computers in Simulation, vol. 79, no. 3, pp. 449-457, 2008.

[16] P. Liu, S. T. Liu, and X. Li, "Adaptive modified function projective synchronization of generaluncertain chaotic complex systems," Physica Scripta, vol. 85, no. 3, Article ID 035005, 2012.

[17] Q. J. Zhang and J. A. Lu, "Passive control and synchronization of hyperchaotic Chen system," Chinese Physics B, vol. 17, no. 2, pp. 492-497, 2008.

[18] D. Q. Wei, X. S. Luo, B. Zhang, and Y. H. Qin, "Controlling chaos in space-clamped FitzHugh-Nagumo neuron by adaptive passive method," Nonlinear Analysis, vol. 11, no. 3, pp. 1752-1759, 2010.

[19] T. Sangpet and S. Kuntanapreeda, "Adaptive synchronization of hyperchaotic systems via passivity feedback control with timevarying gains," Journal of Sound and Vibration, vol. 329, no. 13, pp. 2490-2496, 2010.

[20] D. Q. Wei, B. Zhang, and X. S. Luo, "Adaptive synchronization of chaos in permanent magnet synchronous motors based on passivity theory," Chinese Physics B, vol. 21, no. 3, Article ID 030504, 2012.

[21] G. M. Mahmoud, E. E. Mahmoud, and A. A. Arafa, "Passive control of $n$-dimensional chaotic complex nonlinear systems," Journal of Vibration and Control, vol. 21, no. 3, 2012.

[22] G. M. Mahmoud, E. E. Mahmoud, and M. E. Ahmed, "A hyperchaotic complex Chen system and its dynamics," International Journal of Applied Mathematics \& Statistics, vol. 12, no. D07, pp. 90-100, 2007.

[23] W. Yu, "Passive equivalence of chaos in Lorenz system," IEEE Transactions on Circuits and Systems I-Fundamental Theory and Applications, vol. 46, no. 7, pp. 876-878, 1999.

[24] C. I. Byrnes, A. Isidori, and J. C. Willems, "Passivity, feedback equivalence, and the global stabilization of minimum phase nonlinear systems," IEEE Transactions on Automatic Control, vol. 36, no. 11, pp. 1228-1240, 1991.

[25] G. M. Mahmoud, T. Bountis, and E. E. Mahmoud, "Active control and global synchronization of the complex Chen and Lü systems," International Journal of Bifurcation and Chaos in Applied Sciences and Engineering, vol. 17, no. 12, pp. 4295-4308, 2007.

[26] G. M. Mahmoud, M. E. Ahmed, and E. E. Mahmoud, "Analysis of hyperchaotic complex Lorenz systems," International Journal of Modern Physics C, vol. 19, no. 10, pp. 1477-1494, 2008.

[27] G. M. Mahmoud, E. E. Mahmoud, and M. E. Ahmed, "On the hyperchaotic complex Lü system," Nonlinear Dynamics, vol. 58, no. 4, pp. 725-738, 2009. 


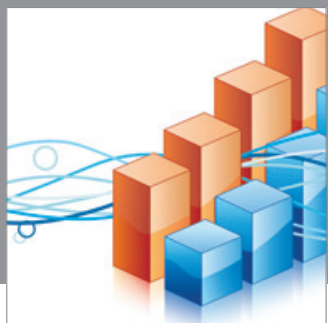

Advances in

Operations Research

mansans

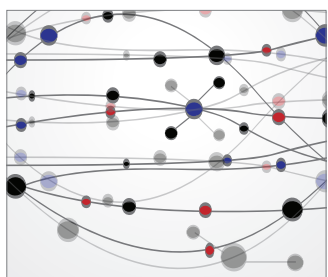

The Scientific World Journal
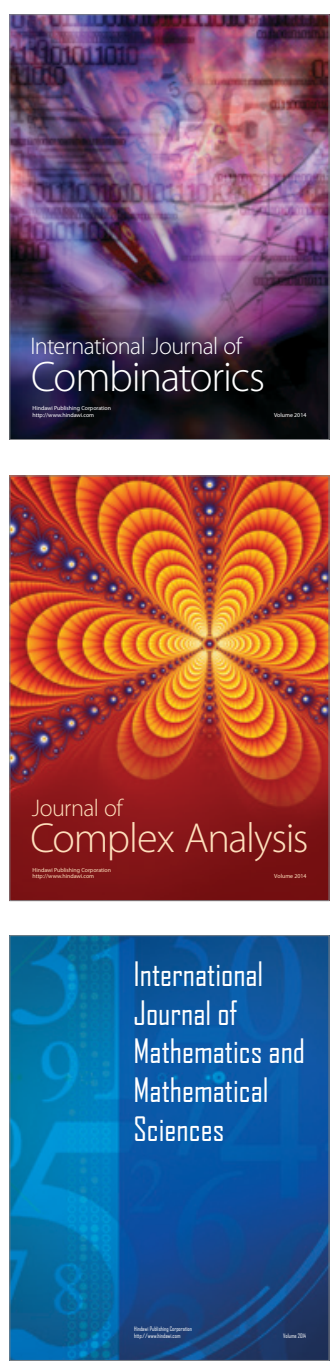
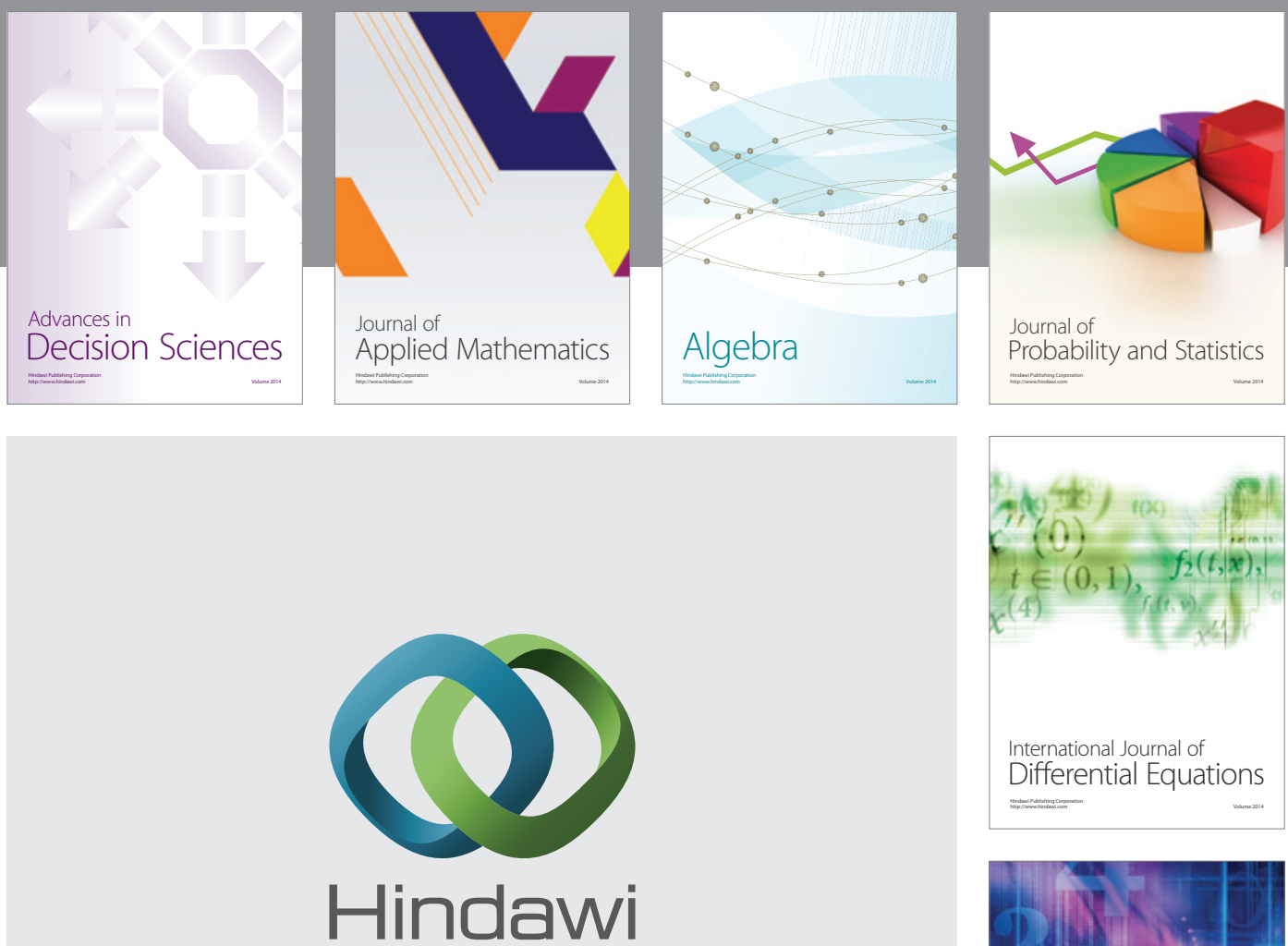

Submit your manuscripts at http://www.hindawi.com
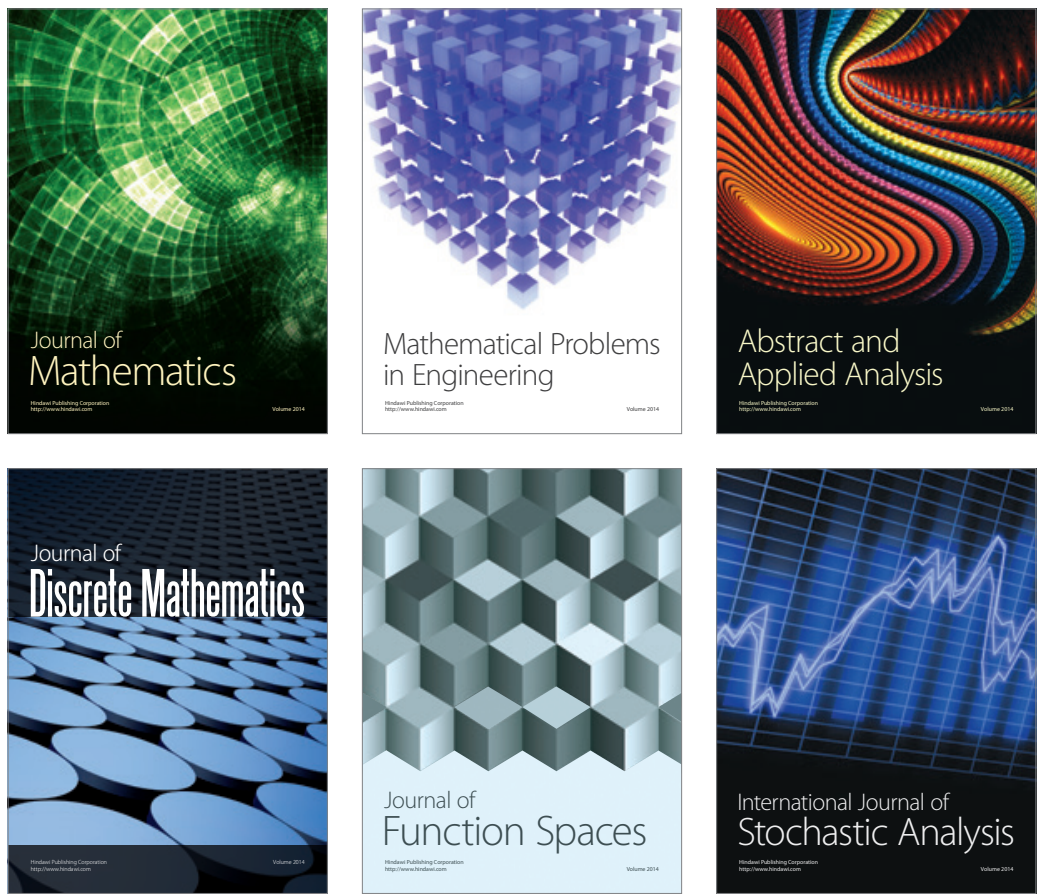

Journal of

Function Spaces

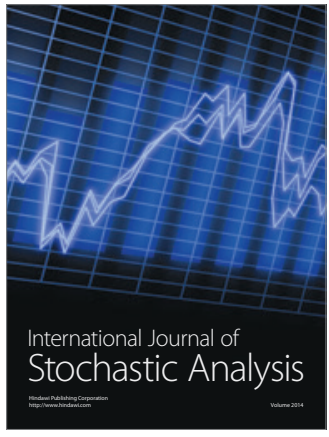

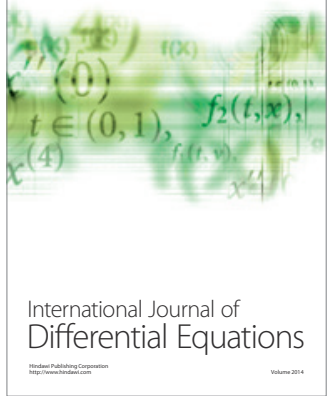
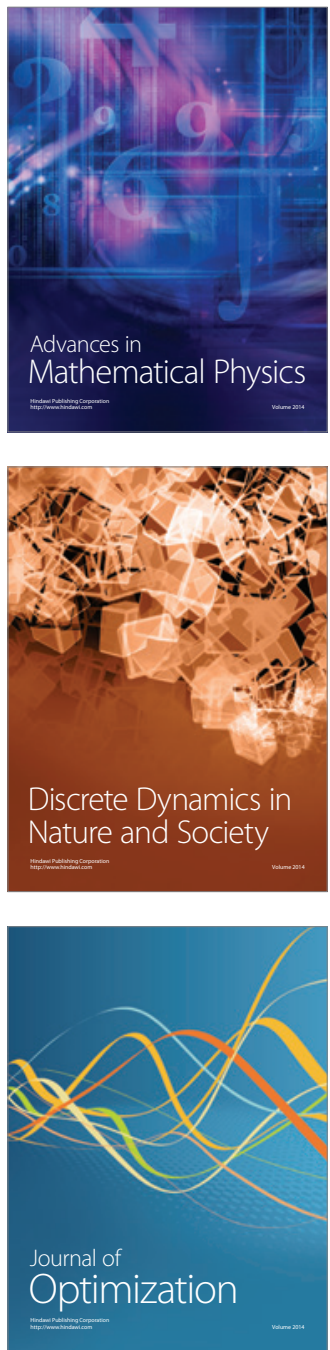